\title{
Viscous Regularization of Delta Shock Wave Solution for a Simplified Chromatography System
}

\author{
Xiumei Li and Chun Shen \\ School of Mathematics and Statistics Science, Ludong University, Yantai, Shandong 264025, China \\ Correspondence should be addressed to Chun Shen; shenchun3641@sina.com
}

Received 7 July 2013; Revised 31 August 2013; Accepted 3 September 2013

Academic Editor: Vladimir Danilov

Copyright (C) 2013 X. Li and C. Shen. This is an open access article distributed under the Creative Commons Attribution License, which permits unrestricted use, distribution, and reproduction in any medium, provided the original work is properly cited.

\begin{abstract}
The delta shock wave for a simplified chromatography system is obtained in the Riemann solution when $-1<v_{-}<0<v_{+}$. In fact, the result in this paper is the reasonable generalization of the result in Sun (2011) with $v_{-}=0<v_{+}$which is exactly the critical situation for $-1<v_{-}<0<v_{+}$. The self-similar viscosity vanishing approach is also used to check the delta shock wave solution.
\end{abstract}

\section{Introduction}

The theory of nonlinear chromatography system is the foundation of chromatography separation process and also plays an important role in the modern industry. Recently, the delta shock wave has been captured numerically and experimentally by Mazzotti et al. [1, 2] in the Riemann solutions for the local equilibrium model of two-component nonlinear chromatography. Since then, extensive attention has been drawn on the formation of delta shock wave solution to all kinds of chromatography systems [3-6].

In [5], Sun considered the Riemann problem for a simplified chromatography system:

$$
\begin{aligned}
& v_{t}+\left(\frac{v}{1+v}\right)_{x}=0, \\
& w_{t}+\left(\frac{w}{1+v}\right)_{x}=0,
\end{aligned}
$$

with the initial data

$$
(v, w)(x, 0)=\left(v_{ \pm}, w_{ \pm}\right), \quad \pm x>0,
$$

where $v_{ \pm}$and $w_{ \pm}$are all given constants. She discovered that a delta shock wave solution also appears in the situation $v_{-}=$ $0<v_{+}$by employing the self-similar viscosity vanishing approach.

In the present paper, we extend the result from the critical situation $v_{-}=0<v_{+}$in [5] to the general situation $-1<v_{-}<$ $0<v_{+}$and discover that the delta shock wave also appears in the Riemann solution to (1) and (2). Actually, in the situation $-1<v_{-}<0<v_{+}$, the Riemann solution to (1) and (2) is a delta shock wave connecting two constant states $\left(v_{+}, w_{+}\right)$ whose propagation speed and strength are, respectively,

$$
\sigma=\frac{1}{\left(1+v_{-}\right)\left(1+v_{+}\right)}, \quad \beta(t)=\frac{\left(w_{-} v_{+}-w_{+} v_{-}\right) t}{\left(1+v_{-}\right)\left(1+v_{+}\right)} .
$$

On the other hand, in the situation $v_{-}=0<v_{+}$in [5], the propagation speed and strength of delta shock wave are, respectively,

$$
\sigma=\frac{1}{1+v_{+}}, \quad \beta(t)=\frac{w_{-} v_{+} t}{1+v_{+}} .
$$

If we let $v_{-} \rightarrow 0+$ in the situation $-1<v_{-}<0<v_{+}$, then the result is identical with that for the situation $v_{-}=0<v_{+}$ in [5]. Thus, one can see that the situation $v_{-}=0<v_{+}$is the critical one, where the delta shock wave can be obtained, and the result in this paper is the reasonable generalization of the result in [5].

Similar to [5], we also consider the following viscous regularization system:

$$
\begin{gathered}
v_{t}+\left(\frac{v}{1+v}\right)_{x}=\varepsilon t v_{x x}, \\
w_{t}+\left(\frac{w}{1+v}\right)_{x}=0,
\end{gathered}
$$


which enables us to see that the delta shock wave is the reasonable choice of the Riemann solution to (1) and (2) when $-1<v_{-}<0<v_{+}$.

In addition, we emphasize that our result here is also reasonable in the sense of chromatography engineer since a simplified chromatography system can also be derived from the other nonlinear chromatography system:

$$
\begin{aligned}
& \partial_{t} u_{1}+\partial_{x}\left(\frac{u_{1}}{1-u_{1}+u_{2}}\right)=0, \\
& \partial_{t} u_{2}+\partial_{x}\left(\frac{u_{2}}{1-u_{1}+u_{2}}\right)=0,
\end{aligned}
$$

by introducing the change of variables

$$
v=u_{2}-u_{1}, \quad w=u_{1}+u_{2},
$$

which is different from the derivation in [5]. Here $u_{1}, u_{2}$ are the nonnegative functions of the variables $(x, t) \in R \times$ $R_{+}$to denote the Langmuir-like and the anti-Langmuir-like components, respectively, which express transformations of the concentrations of two solutes. It is clear that the delta measure appears in $w$ for the system (1) is equivalent to saying that the delta measures appear in both the state variables $u_{1}$ and $u_{2}$ for the system (6). It is easily derived that the strengths of the delta measures in $u_{1}$ and $u_{2}$ are equal to each other. In other words, the phenomena of singular concentrations in the two components $u_{1}$ and $u_{2}$ appear simultaneously for the system (6).

The system (1) is the simplest example for systems of Temple class [7]. In other words, the shock curves coincide with the rarefaction curves in the phase plane. Compared with general systems of conservation laws, well-posed results for systems of Temple class [8] are available for a much larger class of initial data due to the above feature. Furthermore, thanks to the particular structure, wave interactions for the systems of Temple class can also be dealt with completely [9-12], which enables us to solve the Cauchy problem for arbitrary data of bounded variation.

The self-similar viscosity vanishing approach was first proposed by Dafermos [13] to construct Riemann solutions for broad classes of $2 \times 2$ systems that include the equations of isentropic, Lagrangian, and gas dynamics. This method was widely used in [14] for the admissibility of weak solutions of the Riemann problem for hyperbolic systems of conservation laws, and the admissibility criterion was called the wave-fan criterion in [15]. Special attention was also paid in [6, 16-22] to the formation of the delta shock waves in the Riemann solutions for some systems of hyperbolic conservation laws.

There exist numerous excellent papers for the related equations and results about the measure-valued solutions for hyperbolic systems of conservation laws. The well-known examples are the transport equations [21, 23-27] (or called the pressureless Euler equations) and the Chaplygin gas dynamics equations [28, 29] which have been extensively studied. For the other related equations and results, we can refer to [30-35] and the related references therein.

The paper is organized as follows. In Section 2, the Riemann problem of (1) and (2) is considered. In particular, the delta shock wave does occur in the Riemann solution when $-1<v_{-}<0<v_{+}$. In Section 3, we show that there exists a smooth solution for the regularized system (5) with the initial data (2). Then, by letting $\varepsilon \rightarrow 0$, we prove that the limit of the regularized solution is exactly the corresponding Riemann solution to (1) and (2) when $-1<v_{-}<0<v_{+}$.

\section{The Riemann Problem for (1) and (2) in the Phase Space $v>-1$}

In this section, we generalize the Riemann problem for (1) and (2) from the phase space $(v, w) \in[0, \infty) \times(-\infty, \infty)$ in [5] to the phase space $(v, w) \in(-1, \infty) \times(-\infty, \infty)$. Compared with the result in [5] that there are three kinds of the Riemann solutions to (1) and (2) in the phase space $(v, w) \in[0, \infty) \times(-\infty, \infty)$, there exist six kinds of the Riemann solutions to (1) and (2) in the phase space $(v, w) \in$ $(-1, \infty) \times(-\infty, \infty)$ (Figure 1). In what follows, we first depict the properties of (1) and then list the Riemann solutions to (1) and $(2)$ in the phase space $(v, w) \in(-1, \infty) \times(-\infty, \infty)$ case by case. Finally, the generalized Rankine-Hugoniot relations of delta shock wave are derived in detail. We can also refer to $[3,4,12]$ for the related results about the other nonlinear chromatography systems.

The characteristic eigenvalues of (1) are $\lambda_{1}=1 /(1+v)^{2}$ and $\lambda_{2}=1 /(1+v)$, respectively. Thus, it is clear to see that (1) is strictly hyperbolic in the phase space $v>0$ and $-1<v<0$ and is nonstrictly hyperbolic on the line $v=0$. The corresponding right characteristic vectors are $\vec{r}_{1}=(v, w)^{T}$ and $\overrightarrow{r_{2}}=(0,1)^{T}$, respectively. Thus $\lambda_{1}$ is genuinely nonlinear for $v \neq 0$, and $\lambda_{2}$ is always linearly degenerate. Therefore, the associated waves are rarefaction waves $R$ or shock waves $S$ for the characteristic field $\lambda_{1}$ and contact discontinuities $J$ for the characteristic field $\lambda_{2}$. The Riemann invariants along the characteristic fields are $w / v$ and $v$, respectively.

Now we are in a position to deliver the Riemann solutions to (1) and (2) according to the value of $v$ as follows.

(1) If $v_{-}>v_{+}>0$, then the Riemann solution of (1) and (2) can be expressed as $\vec{R}+J$ :

$$
\begin{aligned}
& (v, w)(x, t) \\
& \quad= \begin{cases}\left(v_{-}, w_{-}\right), & x<\lambda_{1}\left(v_{-}\right) t, \\
\left(\sqrt{\frac{t}{x}}-1, \frac{w_{-}}{v_{-}}\left(\sqrt{\frac{t}{x}}-1\right)\right), & \lambda_{1}\left(v_{-}\right) t \leq x \leq \lambda_{1}\left(v_{+}\right) t, \\
\left(v_{+}, \frac{v_{+} w_{-}}{v_{-}}\right), & \lambda_{1}\left(v_{+}\right) t<x<\tau t, \\
\left(v_{+}, w_{+}\right), & x>\tau t,\end{cases}
\end{aligned}
$$

in which the propagation speed of $R$ is $\xi=1 /(1+v)^{2}$ for $v$ from $v_{-}$to $v_{+}$such that $0<\xi<1$ and that of $J$ is $\tau=1 /\left(1+v_{+}\right)$. 


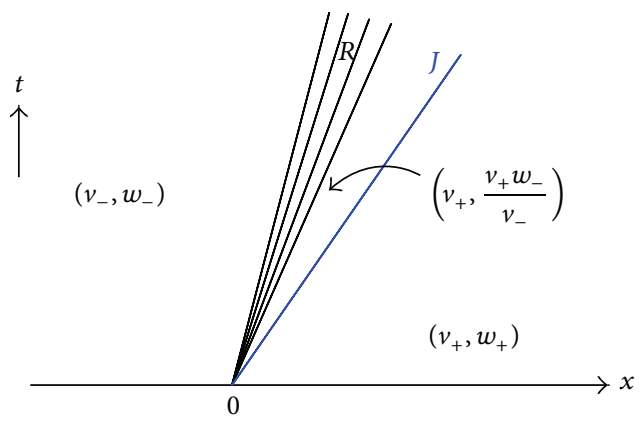

(a) $\vec{R}+J$ when $v_{-}>v_{+}>0$

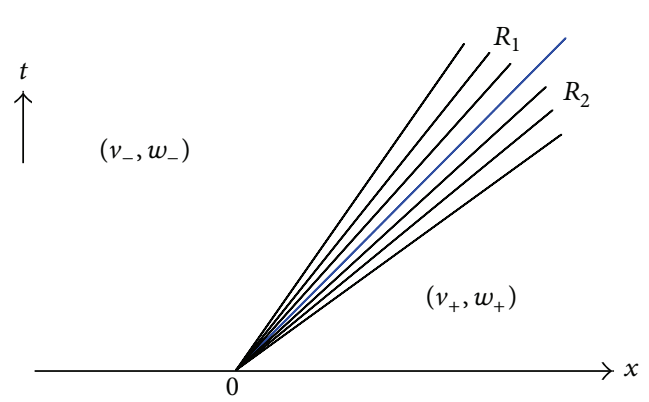

(c) $\vec{R}_{1}+\vec{R}_{2}$ when $-1<v_{+}<0<v_{-}$

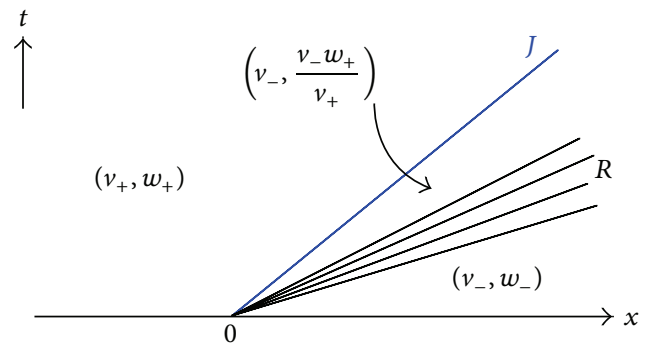

(e) $J+\vec{R}$ when $-1<v_{+}<v_{-}<0$

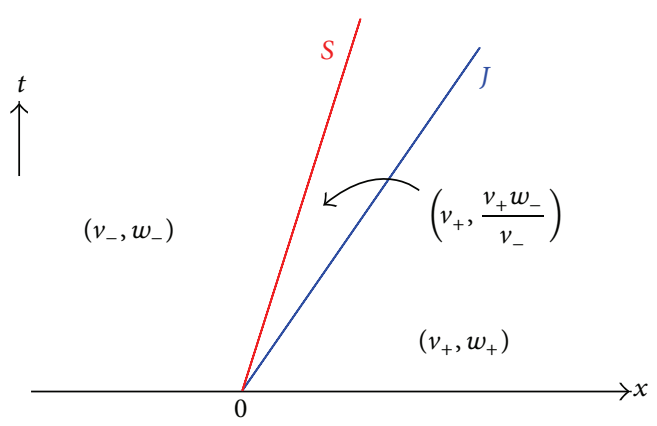

(b) $\vec{S}+J$ when $0<v_{-}<v_{+}$

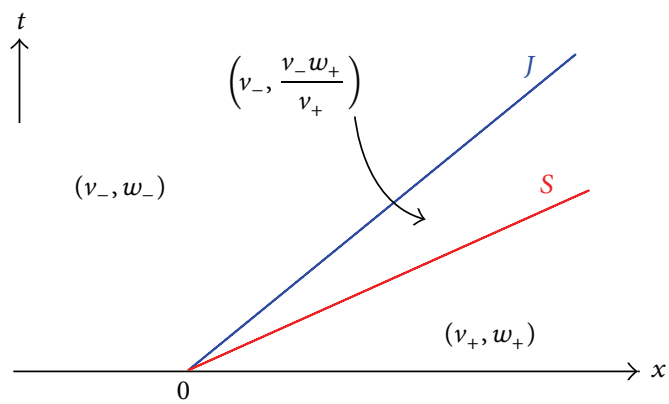

(d) $J+\vec{S}$ when $-1<v_{-}<v_{+}<0$

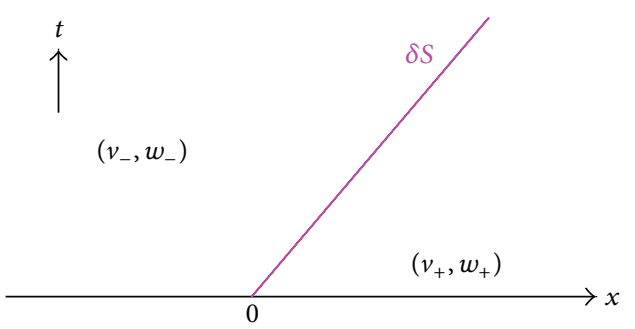

(f) $\delta S$ when $-1<v_{-}<0<v_{+}$

FIgURE 1: The Riemann solutions to (1) and (2) in all the situations.

(2) If $0<v_{-}<v_{+}$, then the Riemann solution of (1) and (2) can be expressed as $\vec{S}+J$ :

$$
(v, w)(x, t)= \begin{cases}\left(v_{-}, w_{-}\right), & x<\sigma t, \\ \left(v_{+}, \frac{v_{+} w_{-}}{v_{-}}\right), & \sigma t<x<\tau t \\ \left(v_{+}, w_{+}\right), & x>\tau t\end{cases}
$$

in which the propagation speed of $S$ is $\sigma=1 /((1+$ $\left.\left.v_{-}\right)\left(1+v_{+}\right)\right)$and that of $J$ is $\tau=1 /\left(1+v_{+}\right)$. It is clear to see that $0<\sigma<\tau<1$.

(3) If $-1<v_{+}<0<v_{-}$, then the Riemann solution of (1) and (2) can be expressed as $\vec{R}_{1}+\vec{R}_{2}$ : $(v, w)(x, t)$

$$
= \begin{cases}\left(v_{-}, w_{-}\right), & x<\lambda_{1}\left(v_{-}\right) t, \\ \left(\sqrt{\frac{t}{x}}-1, \frac{w_{-}}{v_{-}}\left(\sqrt{\frac{t}{x}}-1\right)\right), & \lambda_{1}\left(v_{-}\right) t \leq x<t, \\ \left(\sqrt{\frac{t}{x}}-1, \frac{w_{+}}{v_{+}}\left(\sqrt{\frac{t}{x}}-1\right)\right), & t<x \leq \lambda_{1}\left(v_{+}\right) t, \\ \left(v_{+}, w_{+}\right), & x>\lambda_{1}\left(v_{+}\right) t .\end{cases}
$$

It should be emphasized that the state $(v, w)$ in $\vec{R}_{1}$ is determined by $w / v=w_{-} / v_{-}$which is on the left of the line $x=t$; otherwise, the state $(v, w)$ in $\vec{R}_{2}$ is determined by $w / v=w_{+} / v_{+}$which is on the right of the line $x=t$.

(4) If $-1<v_{-}<v_{+}<0$, then the Riemann solution of (1) and (2) can be expressed as $J+\vec{S}$ : 


$$
(v, w)(x, t)= \begin{cases}\left(v_{-}, w_{-}\right), & x<\tau t, \\ \left(v_{-}, \frac{v_{-} w_{+}}{v_{+}}\right), & \tau t<x<\sigma t \\ \left(v_{+}, w_{+}\right), & x>\sigma t,\end{cases}
$$

in which the propagation speed of $J$ is $\tau=1 /\left(1+v_{-}\right)$ and that of $S$ is $\sigma=1 /\left(1+v_{-}\right)\left(1+v_{+}\right)$. Here we notice that $1<\tau<\sigma$.

(5) If $-1<v_{+}<v_{-}<0$, then the Riemann solution of (1) and (2) can be expressed as $J+\vec{R}$ :

$(v, w)(x, t)$

$$
= \begin{cases}\left(v_{-}, w_{-}\right), & x<\tau t, \\ \left(v_{-}, \frac{v_{-} w_{+}}{v_{+}}\right), & \tau t<x<\lambda_{1}\left(v_{-}\right) t, \\ \left(\sqrt{\frac{t}{x}}-1, \frac{w_{+}}{v_{+}}\left(\sqrt{\frac{t}{x}}-1\right)\right), & \lambda_{1}\left(v_{-}\right) t \leq x \leq \lambda_{1}\left(v_{+}\right) t, \\ \left(v_{+}, w_{+}\right), & x>\lambda_{1}\left(v_{+}\right) t,\end{cases}
$$

in which the propagation speed of $J$ is $\tau=1 /\left(1+v_{-}\right)>$ 1 and that of $R$ is $\xi=1 /(1+v)^{2}>1$ for $v$ from $v_{-}$to $v_{+}$.

(6) For the case $-1<v_{-}<0<v_{+}$, motivated by the result in [22], we consider that the Riemann solution to (1) and (2) is a delta shock wave $\delta S$ connecting the two constant states $\left(v_{-}, w_{-}\right)$and $\left(v_{+}, w_{+}\right)$. In order to deal with it, we need the following definition of a delta shock wave solution in the framework introduced in [36] and used in [12, 37].

Let us suppose that $\Gamma=\left\{\gamma_{i} \mid i \in I\right\}$ is a graph in the closed upper half-plane $\{(x, t) \mid(x, t) \in(-\infty, \infty) \times[0, \infty)\}$, containing Lipschitz continuous arcs $\gamma_{i}$ with $i \in I$, in which $I$ is a finite index set. Let us also suppose that $I_{0}$ is the subset of $I$ containing all indices of arcs linking to the $x$-axis and $\Gamma_{0}=\left\{x_{k}^{0} \mid k \in I_{0}\right\}$ is the set of initial points of the arcs $\gamma_{k}$ with $k \in I_{0}$.

Definition 1. Let $(v, w)$ be a pair of distributions, where $w$ is represented in the form

$$
w(x, t)=\widehat{w}(x, t)+\alpha(x, t) \delta(\Gamma),
$$

in which $v, \widehat{w} \in L^{\infty}\left(R \times R_{+}\right)$and the singular part should be defined by

$$
\alpha(x, t) \delta(\Gamma)=\sum_{i \in I} \alpha_{i}(x, t) \delta\left(\gamma_{i}\right)
$$

Let us consider the initial data of the form

$$
(v, w)(x, 0)=\left(v_{0}(x), \widehat{w}_{0}(x)+\sum_{k \in I_{0}} \alpha_{k}\left(x_{k}^{0}, 0\right) \delta\left(x-x_{k}^{0}\right)\right),
$$

in which $v_{0}, \widehat{w}_{0} \in L^{\infty}(R)$; then the earlier pair of distributions $(v, w)$ are called a generalized delta shock wave solution of the simplified chromatography system (1) with the initial data (15) if the following integral identities

$$
\begin{array}{r}
\int_{R_{+}} \int_{R}\left(v \psi_{t}+\frac{v}{1+v} \psi_{x}\right) d x d t+\int_{R} v_{0}(x) \psi(x, 0) d x=0, \\
\int_{R_{+}} \int_{R}\left(\widehat{w} \psi_{t}+\frac{\widehat{w}}{1+v} \psi_{x}\right) d x d t+\sum_{i \in I} \int_{\gamma_{i}} \alpha_{i}(x, t) \frac{\partial \psi(x, t)}{\partial l} \\
+\int_{R} \widehat{w}_{0}(x) \psi(x, 0) d x+\sum_{k \in I_{0}} \alpha_{k}\left(x_{k}^{0}, 0\right) \psi\left(x_{k}^{0}, 0\right)=0
\end{array}
$$

hold for all test functions $\psi \in C_{c}^{\infty}\left(R \times R_{+}\right)$, in which $\partial \psi(x, t) / \partial l$ denotes the tangential derivative of a function $\psi$ on the graph $\gamma_{i}$ and $\int_{\gamma_{i}}$ denotes the line integral along the arc $\gamma_{i}$.

With the above definition, similar to that in [5], we can also construct the Riemann solution to (1) and (2) for the situation $-1<v_{-}<0<v_{+}$in the following theorem.

Theorem 2. For the case $-1<v_{-}<0<v_{+}$, the Riemann problem (1) and (2) has the piecewise smooth Riemann solution in the form

$$
(v, w)(x, t)= \begin{cases}\left(v_{-}, w_{-}\right), & x<\sigma t \\ \left(v_{\delta}, \beta(t) \delta(x-\sigma t)\right), & x=\sigma t \\ \left(v_{+}, w_{+}\right), & x>\sigma t\end{cases}
$$

where

$$
\begin{gathered}
v_{\delta}=v_{-}+v_{+}+v_{-} v_{+}, \quad \sigma=\frac{1}{1+v_{\delta}}=\frac{1}{\left(1+v_{-}\right)\left(1+v_{+}\right)}, \\
\beta(t)=\frac{\left(w_{-} v_{+}-w_{+} v_{-}\right) t}{\left(1+v_{-}\right)\left(1+v_{+}\right)}
\end{gathered}
$$

The measure solution (17) with (18) also satisfies the generalized Rankine-Hugoniot condition as follows:

$$
\begin{gathered}
\frac{d x}{d t}=\sigma, \\
\frac{d \beta(t)}{d t}=\sigma[w]-\left[\frac{w}{1+v}\right], \\
\sigma[v]=\left[\frac{v}{1+v}\right] .
\end{gathered}
$$

Proof. Let us check that the $\delta$-measure solution (17) with (18) obeys (1) in the sense of distributions. In other words, we only need to check that (17) with (18) satisfies

$$
\begin{aligned}
& \int_{0}^{\infty} \int_{-\infty}^{\infty}\left(v \psi_{t}+\frac{v}{1+v} \psi_{x}\right) d x d t=0 \\
& \int_{0}^{\infty} \int_{-\infty}^{\infty}\left(w \psi_{t}+\frac{w}{1+v} \psi_{x}\right) d x d t=0
\end{aligned}
$$


Actually, we can verify the first equation in (20) as follows:

$$
\begin{aligned}
& \int_{0}^{\infty} \int_{-\infty}^{\infty}\left(v \psi_{t}+\frac{v}{1+v} \psi_{x}\right) d x d t \\
& =\int_{0}^{\infty} \int_{-\infty}^{\sigma t}\left(v_{-} \psi_{t}+\frac{v_{-}}{1+v_{-}} \psi_{x}\right) d x d t \\
& +\int_{0}^{\infty} \int_{\sigma t}^{\infty}\left(v_{+} \psi_{t}+\frac{v_{+}}{1+v_{+}} \psi_{x}\right) d x d t \\
& =\int_{0}^{\infty} \int_{-\infty}^{0} v_{-} \psi_{t} d x d t+\int_{0}^{\infty} \int_{0}^{\sigma t} v_{-} \psi_{t} d x d t \\
& +\int_{0}^{\infty} \int_{-\infty}^{\sigma t} \frac{v_{-} \psi_{x}}{1+v_{-}} d x d t \\
& +\int_{0}^{\infty} \int_{\sigma t}^{\infty}\left(v_{+} \psi_{t}+\frac{v_{+}}{1+v_{+}} \psi_{x}\right) d x d t \\
& =\int_{0}^{\infty} \int_{x / \sigma}^{+\infty} v_{-} \psi_{t} d t d x+\int_{0}^{\infty} \int_{-\infty}^{\sigma t} \frac{v_{-} \psi_{x}}{1+v_{-}} d x d t \\
& +\int_{0}^{\infty} \int_{0}^{x / \sigma} v_{+} \psi_{t} d t d x+\int_{0}^{\infty} \int_{\sigma t}^{\infty} \frac{v_{+} \psi_{x}}{1+v_{+}} d x d t \\
& =-\int_{0}^{\infty} v_{-} \psi\left(x, \frac{x}{\sigma}\right) d x+\int_{0}^{\infty} \frac{v_{-}}{1+v_{-}} \psi(\sigma t, t) d t \\
& +\int_{0}^{\infty} v_{+} \psi\left(x, \frac{x}{\sigma}\right) d t-\int_{0}^{\infty} \frac{v_{+}}{1+v_{+}} \psi(\sigma t, t) d t \\
& =0 \text {, }
\end{aligned}
$$

in which the fact that $\psi(x, t)$ is compactly supported in the region $R \times R_{+}$has been used.

Consequently, for the second equation of (20), we have

$$
\begin{aligned}
\int_{0}^{\infty} \int_{-\infty}^{\infty}\left(w \psi_{t}+\frac{w}{1+v} \psi_{x}\right) d x d t \\
=\int_{0}^{\infty} \int_{-\infty}^{\sigma t}\left(w_{-} \psi_{t}+\frac{w_{-} \psi_{x}}{1+v_{-}}\right) d x d t \\
+\int_{0}^{\infty} \int_{\sigma t}^{\infty}\left(w_{+} \psi_{t}+\frac{w_{+} \psi_{x}}{1+v_{+}}\right) d x d t \\
+\int_{0}^{\infty} \beta(t)\left(\psi_{t}(\sigma t, t)+\sigma \psi_{x}(\sigma t, t)\right) d t \\
=\int_{0}^{\infty} \int_{0}^{\sigma t} w_{-} \psi_{t} d x d t+\int_{0}^{\infty} \int_{-\infty}^{\sigma t} \frac{w_{-} \psi_{x}}{1+v_{-}} d x d t \\
\quad+\int_{0}^{\infty} \int_{\sigma t}^{\infty} w_{+} \psi_{t} d x d t+\int_{0}^{\infty} \int_{\sigma t}^{\infty} \frac{w_{+} \psi_{x}}{1+v_{+}} d x d t \\
+\int_{0}^{\infty} \beta(t)\left(\psi_{t}(\sigma t, t)+\sigma \psi_{x}(\sigma t, t)\right) d t \\
=\int_{0}^{\infty}\left(w_{+}-w_{-}\right) \psi\left(x, \frac{x}{\sigma}\right) d x
\end{aligned}
$$

$$
\begin{aligned}
& +\int_{0}^{\infty}\left(\frac{w_{-}}{1+v_{-}}-\frac{w_{+}}{1+v_{+}}\right) \psi(\sigma t, t) d t \\
& +\int_{0}^{\infty} \beta(t) d \psi(\sigma t, t) \\
& =\left[\frac{w_{+}-w_{-}}{\left(1+v_{-}\right)\left(1+v_{+}\right)}+\frac{w_{-}}{1+v_{-}}-\frac{w_{+}}{1+v_{+}}-\beta^{\prime}(t)\right] \\
& \quad \times \int_{0}^{\infty} \psi(\sigma t, t) d t \\
& =0,
\end{aligned}
$$

in which the changes of variables and integration by parts have been used.

Through the earlier verification, we can conclude that (17) with (18) is indeed the piecewise smooth Riemann solution to (1) and (2) in the sense of distributions.

Remark 3. If we take the limit $v_{-} \rightarrow 0$ in the Riemann solution (17) with (18), then we can obtain the result which is identical with that in [5]. In other words, the Riemann solution to (1) and (2) for $0=v_{-}<v_{+}$can be seen as the critical situation for $-1<v_{-}<0<v_{+}$. Thus, the result in [5] can be seen as the special situation in this paper.

Remark 4. If we take the limit $v_{-} \rightarrow-1$ in the Riemann solution (17) with (18), then both the propagation speed and strength of the delta shock wave will tend to $+\infty$. In fact, both $\lambda_{1}=1 /(1+v)^{2}$ and $\lambda_{2}=1 /(1+v)$ tend to $+\infty$ if we let $v \rightarrow-1$ on the right. Thus, the line $v=-1$ in the phase space $(v, w)$ can be seen as a singular curve.

Remark 5. The Riemann solution to (1) and (2) can be constructed simply if we take the Riemann initial data (2) in the phase space $(v, w) \in(-\infty,-1) \times(-\infty, \infty)$. However, it is difficult to construct the global Riemann solution to (1) and (2) if we require the Riemann initial data (2) in the phase space $(v, w) \in(-\infty, \infty) \times(-\infty, \infty)$. In this situation, we at least need to make some criterions on the line $v=-1$.

\section{Viscous Regularization of Delta Shock Wave Solution}

In this section, we are only interested in the viscous regularization of delta shock wave solution to the Riemann problem (1) and (2). Thus, we confine our attention only to the situation $-1<v_{-}<0<v_{+}$.

Performing the self-similar transformation $\xi=x / t$, we get the boundary value problem:

$$
\begin{gathered}
-\xi v_{\xi}+\left(\frac{v}{1+v}\right)_{\xi}=\varepsilon v_{\xi \xi}, \\
-\xi w_{\xi}+\left(\frac{w}{1+v}\right)_{\xi}=0,
\end{gathered}
$$

with the boundary conditions

$$
(v, w)( \pm \infty)=\left(v_{ \pm}, w_{ \pm}\right) .
$$


By observing (23), we know that the solution of the first equation in (23) does not rely on the second one in (23). So, we can first consider the first equation in (23), namely,

$$
\begin{gathered}
-\xi v_{\xi}+\left(\frac{v}{1+v}\right)_{\xi}=\varepsilon v_{\xi \xi}, \\
v( \pm \infty)=v_{ \pm} .
\end{gathered}
$$

With the similar deduction and calculation in Theorem 4.1 in [5], we can also obtain the same conclusion as follows.

Theorem 6. For each fixed $\varepsilon>0$, there exists a unique and monotonic smooth solution $v^{\varepsilon}(\xi)$ of the boundary value problem (25) for $-1<v_{-}<0<v_{+}$.

Now, we turn our attention to the second equation in (23) and consider the existence of the solution $w(\xi)$. Based on Theorem 6, also with the similar deduction and calculation in Theorem 4.2 in [5], we can obtain the following theorem.

Theorem 7. For each fixed $\varepsilon>0$, assume that $w(\xi)$ is a weak solution of the following boundary value problem:

$$
\begin{gathered}
-\xi w_{\xi}+\left(\frac{w}{1+v^{\varepsilon}}\right)_{\xi}=0, \\
w( \pm \infty)=w_{ \pm},
\end{gathered}
$$

where $v^{\varepsilon}(\xi)$ is the solution of (25).

Then, one has $w^{\varepsilon}(\xi) \in L^{1}(-\infty,+\infty)$ which can be expressed as

$w^{\varepsilon}(\xi)$

$$
= \begin{cases}w_{-} \exp \left(\int_{-\infty}^{\xi} \frac{\dot{v}^{\varepsilon}(s)}{1+v^{\varepsilon}(s)-s\left(1+v^{\varepsilon}(s)\right)^{2}}\right) d s \quad \text { for } \xi<\xi_{\alpha}^{\varepsilon}, \\ w_{+} \exp \left(\int_{\xi}^{+\infty} \frac{-\dot{v}^{\varepsilon}(s)}{1+v^{\varepsilon}(s)-s\left(1+v^{\varepsilon}(s)\right)^{2}}\right) d s \quad \text { for } \xi>\xi_{\alpha}^{\varepsilon}\end{cases}
$$

where $\xi_{\alpha}^{\varepsilon}$ is the unique fixed point of $\xi=1 /\left(1+v^{\varepsilon}(\xi)\right)$.

It follows from Theorem 6 that $v^{\varepsilon}(\xi)$ is strictly monotonically increasing with respect to $\xi$ when $-1<v_{-}<$ $0<v_{+}$, which enables us to see that both $1 /\left(1+v^{\varepsilon}(\xi)\right)$ and $1 /\left(1+v^{\varepsilon}(\xi)\right)^{2}$ are strictly monotonically decreasing with respect to $\xi$ when $-1<v_{-}<0<v_{+}$. Thus, there exist unique fixed points of the following equations, and we use $\xi_{\alpha}^{\varepsilon}$ and $\xi_{\beta}^{\varepsilon}$ to express as

$$
\begin{gathered}
\xi_{\alpha}^{\varepsilon}=\frac{1}{1+v^{\varepsilon}\left(\xi_{\alpha}^{\varepsilon}\right)}, \\
\xi_{\beta}^{\varepsilon}=\frac{1}{\left(1+v^{\varepsilon}\left(\xi_{\beta}^{\varepsilon}\right)\right)^{2}},
\end{gathered}
$$

respectively. Consequently, we take the limits $\xi_{\alpha}=\lim _{\varepsilon \rightarrow 0^{+}} \xi_{\alpha}^{\varepsilon}$ and $\xi_{\beta}=\lim _{\varepsilon \rightarrow 0^{+}} \xi_{\beta}^{\varepsilon}$, which will play very critical roles in the following discussion.
Lemma 8. For any $\eta>0$, draws the conclusion that

$$
\lim _{\varepsilon \rightarrow 0^{+}} v^{\varepsilon}(\xi)= \begin{cases}v_{-}, & \text {for } \xi<\xi_{\beta}-\eta, \\ v_{+}, & \text {for } \xi>\xi_{\beta}+\eta\end{cases}
$$

is in uniform in the earlier intervals.

Proof. It is easy to take $\varepsilon>0$ sufficiently small such that $\xi_{\beta}^{\varepsilon}<$ $\xi_{\beta}+\eta / 4$ for $\xi_{\beta}=\lim _{\varepsilon \rightarrow 0^{+}} \xi_{\beta}^{\varepsilon}$. If we take $\xi_{1}=\xi_{\beta}+\eta / 2$, then it is clear that $\xi_{\beta}^{\varepsilon}<\xi_{1}-\eta / 4$. From Theorem 6 , we know that $\dot{v}^{\varepsilon}(\xi)>0$ if $-1<v_{-}<0<v_{+}$. Then (25) can be written as

$$
\frac{1}{\varepsilon}\left(-\xi+\frac{1}{\left(1+v^{\varepsilon}(\xi)\right)^{2}}\right)=\frac{\ddot{v}^{\varepsilon}(\xi)}{\dot{v}^{\varepsilon}(\xi)} .
$$

By integrating $(31)$ over $\left(\xi_{1}, \xi\right)$, we arrive at

$$
\dot{v}^{\varepsilon}(\xi)=\dot{v}^{\varepsilon}\left(\xi_{1}\right) \exp \int_{\xi_{1}}^{\xi} \frac{1}{\varepsilon}\left(-s+\frac{1}{\left(1+v^{\varepsilon}(s)\right)^{2}}\right) d s .
$$

Integrating (32) over $\left(\xi_{1},+\infty\right)$, we get

$$
\begin{aligned}
v_{+} & -v^{\varepsilon}\left(\xi_{1}\right) \\
& =\dot{v}^{\varepsilon}\left(\xi_{1}\right) \int_{\xi_{1}}^{+\infty} \exp \left(\int_{\xi_{1}}^{\xi} \frac{1}{\varepsilon}\left(-s+\frac{1}{\left(1+v^{\varepsilon}(s)\right)^{2}}\right) d s\right) d \xi .
\end{aligned}
$$

Because $v^{\varepsilon}(\xi)$ is monotonically increasing in the real axis, we have $\dot{v}^{\varepsilon}\left(\xi_{1}\right) \geq 0$ and $v_{-} \leq v^{\varepsilon}(s) \leq v_{+}$. Thus, one can conclude that

$$
\begin{aligned}
v_{+} & -v_{-} \\
& \geq v_{+}-v^{\varepsilon}\left(\xi_{1}\right) \\
& =\dot{v}^{\varepsilon}\left(\xi_{1}\right) \int_{\xi_{1}}^{+\infty} \exp \left(\int_{\xi_{1}}^{\xi} \frac{1}{\varepsilon}\left(-s+\frac{1}{\left(1+v^{\varepsilon}(s)\right)^{2}}\right) d s\right) d \xi \\
& \geq \dot{v}^{\varepsilon}\left(\xi_{1}\right) \int_{\xi_{1}}^{+\infty} \exp \frac{1}{\varepsilon}\left(-\frac{1}{2}\left(\xi-\xi_{1}\right)\left(\xi+\xi_{1}\right)+\frac{\xi-\xi_{1}}{\left(1+v_{+}\right)^{2}}\right) d \xi \\
& =\dot{v}^{\varepsilon}\left(\xi_{1}\right) \int_{0}^{+\infty} \exp \frac{1}{\varepsilon}\left(-\frac{1}{2} \zeta^{2}+\zeta\left(\frac{1}{\left(1+v_{+}\right)^{2}}-\xi_{1}\right)\right) d \zeta \\
& \geq \dot{v}^{\varepsilon}\left(\xi_{1}\right) \cdot \varepsilon C_{1}
\end{aligned}
$$

for $0<\varepsilon<1$ and $C_{1}$ is a constant independent of $\varepsilon$, in which the change of variables $\zeta=\xi-\xi_{1}$ has been used. So, we can get $0 \leq \dot{v}^{\varepsilon}\left(\xi_{1}\right) \leq\left(v_{+}-v_{-}\right) /\left(\varepsilon C_{1}\right)$. Together with (32), we know that

$$
\dot{v}^{\varepsilon}(\xi) \leq \frac{v_{+}-v_{-}}{\varepsilon C_{1}} \exp \left(\int_{\xi_{1}}^{\xi} \frac{1}{\varepsilon}\left(-s+\frac{1}{\left(1+v^{\varepsilon}(s)\right)^{2}}\right) d s\right) .
$$

In view of (29), we have

$$
\begin{aligned}
-s+ & \frac{1}{\left(1+v^{\varepsilon}(s)\right)^{2}} \\
& =\frac{1}{\left(1+v^{\varepsilon}(s)\right)^{2}}-\frac{1}{\left(1+v^{\varepsilon}\left(\xi_{\beta}^{\varepsilon}\right)\right)^{2}}-\left(s-\xi_{\beta}^{\varepsilon}\right),
\end{aligned}
$$


noticing that $\xi_{\beta}^{\varepsilon}+\eta / 4<\xi_{1}<s$, by applying the mean value theorem, and then there exists $\xi_{2} \in\left(\xi_{\beta}^{\varepsilon}, s\right)$ such that

$$
-s+\frac{1}{\left(1+v^{\varepsilon}(s)\right)^{2}}=\left(\frac{-2 \dot{v}^{\varepsilon}\left(\xi_{2}\right)}{\left(1+v^{\varepsilon}\left(\xi_{2}\right)\right)^{3}}-1\right)\left(s-\xi_{\beta}^{\varepsilon}\right) \leq-\frac{\eta}{4} .
$$

So, we get the following inequality:

$$
\dot{v}^{\varepsilon}(\xi) \leq \frac{v_{+}-v_{-}}{\varepsilon C_{1}} \exp \left(-\frac{\eta}{4 \varepsilon}\left(\xi-\xi_{1}\right)\right),
$$

which means that $\dot{v}^{\varepsilon}(\xi) \rightarrow 0$ uniformly on $\xi>\xi_{\beta}+\eta$.

For any $\xi>\xi_{\beta}+\eta$, we have

$$
v_{+}-v^{\varepsilon}(\xi)=\int_{\xi}^{+\infty} \dot{v}^{\varepsilon}(s) d s .
$$

Obviously, we know that $\lim _{\varepsilon \rightarrow 0^{+}} v^{\varepsilon}(\xi)=v_{+}$uniformly on $\xi>\xi_{\beta}+\eta$ in view of (38).

Now, we try to prove that $\lim _{\varepsilon \rightarrow 0^{+}} v^{\varepsilon}(\xi)=v_{-}$uniformly on $\xi<\xi_{\beta}-\eta$. Similarly, it is easy to take $\varepsilon>0$ sufficiently small such that $\xi_{\beta}^{\varepsilon}>\xi_{\beta}-\eta / 4$. If we take $\xi_{3}=\xi_{\beta}-\eta / 2$, then we also have $\xi_{\beta}^{\varepsilon}>\xi_{3}+\eta / 4$. By integrating (25) over $\left(\xi, \xi_{3}\right)$, we have

$$
\dot{v}^{\varepsilon}(\xi)=\dot{v}^{\varepsilon}\left(\xi_{3}\right) \exp \int_{\xi_{3}}^{\xi} \frac{1}{\varepsilon}\left(-s+\frac{1}{\left(1+v^{\varepsilon}(s)\right)^{2}}\right) d s .
$$

Integrating (25) over $\left(-\infty, \xi_{3}\right)$, we have

$$
\begin{aligned}
& v^{\varepsilon}\left(\xi_{3}\right)-v_{-} \\
& \quad=\dot{v}^{\varepsilon}\left(\xi_{3}\right) \int_{-\infty}^{\xi_{3}} \exp \left(\int_{\xi_{3}}^{\xi} \frac{1}{\varepsilon}\left(-s+\frac{1}{\left(1+v^{\varepsilon}(s)\right)^{2}}\right) d s\right) d \xi .
\end{aligned}
$$

Thus, we get the following result:

$$
\begin{aligned}
v_{+} & -v_{-} \\
& \geq v^{\varepsilon}\left(\xi_{3}\right)-v_{-} \\
& =\dot{v}^{\varepsilon}\left(\xi_{3}\right) \int_{-\infty}^{\xi_{3}} \exp \left(\int_{\xi_{3}}^{\xi} \frac{1}{\varepsilon}\left(-s+\frac{1}{\left(1+v^{\varepsilon}(s)\right)^{2}}\right) d s\right) d \xi \\
& \geq \dot{v}^{\varepsilon}\left(\xi_{3}\right) \int_{-\infty}^{\xi_{3}} \exp \frac{1}{\varepsilon}\left(-\frac{1}{2}\left(\xi-\xi_{3}\right)\left(\xi+\xi_{3}\right)+\frac{\xi-\xi_{3}}{\left(1+v_{-}\right)^{2}}\right) d \xi \\
& =\dot{v}^{\varepsilon}\left(\xi_{3}\right) \int_{-\infty}^{0} \exp \frac{1}{\varepsilon}\left(-\frac{1}{2} \zeta^{2}+\zeta\left(\frac{1}{\left(1+v_{-}\right)^{2}}-\xi_{3}\right)\right) d \zeta \\
& \geq \dot{v}^{\varepsilon}\left(\xi_{3}\right) \cdot \varepsilon C_{2}
\end{aligned}
$$

for $0<\varepsilon<1$ and $C_{2}$ is a constant independent of $\varepsilon$, in which the change of variables $\zeta=\xi-\xi_{3}$ has also been used. Thus we have $\dot{v}^{\varepsilon}\left(\xi_{3}\right) \leq\left(v_{+}-v_{-}\right) /\left(\varepsilon C_{2}\right)$, together with (40), and we also know that

$$
\dot{v}^{\varepsilon}(\xi) \leq \frac{v_{+}-v_{-}}{\varepsilon C_{2}} \exp \left(\int_{\xi_{3}}^{\xi} \frac{1}{\varepsilon}\left(-s+\frac{1}{\left(1+v^{\varepsilon}(s)\right)^{2}}\right) d s\right) .
$$

Similarly, by applying the mean value theorem, then there exists $\xi_{4} \in\left(s, \xi_{\beta}^{\varepsilon}\right)$ such that

$$
-s+\frac{1}{\left(1+v^{\varepsilon}(s)\right)^{2}}=\left(\frac{-2 \dot{v}^{\varepsilon}\left(\xi_{4}\right)}{\left(1+v^{\varepsilon}\left(\xi_{4}\right)\right)^{3}}-1\right)\left(s-\xi_{\beta}^{\varepsilon}\right) \geq \frac{\eta}{4} .
$$

Combining (43) together with (44), we get

$$
\dot{v}^{\varepsilon}(\xi) \leq \frac{v_{+}-v_{-}}{\varepsilon C_{2}} \exp \left(-\frac{\eta}{4 \varepsilon}\left(\xi_{3}-\xi\right)\right),
$$

which implies that $\dot{v}^{\varepsilon}(\xi) \rightarrow 0$ uniformly on $\xi<\xi_{\beta}-\eta$.

Thus, for any $\xi<\xi_{\beta}-\eta$, we have

$$
v^{\varepsilon}(\xi)-v_{-}=\int_{-\infty}^{\xi} \dot{v}^{\varepsilon}(s) d s .
$$

Obviously, we can see that $\lim _{\varepsilon \rightarrow 0^{+}} v^{\varepsilon}(\xi)=v_{-}$uniformly on $\xi<\xi_{\beta}-\eta$ in view of (45).

Lemma 9. Let $\xi_{\alpha}$ and $\xi_{\beta}$ be defined as above. Then, one has

$$
\xi_{\alpha}=\xi_{\beta}=\frac{1}{\left(1+v_{-}\right)\left(1+v_{+}\right)} .
$$

Proof. Take any $\phi(\xi) \in C_{0}^{\infty}\left(\xi_{1}, \xi_{2}\right)$ where $\xi_{1}<\xi_{\beta}<\xi_{2}$. It follows from (25) that

$$
\int_{\xi_{1}}^{\xi_{2}} \varepsilon v_{\xi \xi} \cdot \phi(\xi) d \xi=\int_{\xi_{1}}^{\xi_{2}}\left(-\xi v_{\xi}+\left(\frac{v}{1+v}\right)_{\xi}\right) \phi(\xi) d \xi .
$$

By employing integration by parts and applying the fact that $\phi$ is compactly supported in $\left(\xi_{1}, \xi_{2}\right)$, we have

$$
\int_{\xi_{1}}^{\xi_{2}} \varepsilon v \phi^{\prime \prime}(\xi) d \xi=\int_{\xi_{1}}^{\xi_{2}}\left(v\left(\phi(\xi)+\xi \phi^{\prime}(\xi)\right)-\frac{v}{1+v} \phi^{\prime}(\xi)\right) d \xi .
$$

Taking the limit $\varepsilon \rightarrow 0$ in (49), it can be obtained from Lemma 8 that

$$
\begin{aligned}
& \int_{\xi_{1}}^{\xi_{\beta}}\left(v_{-}\left(\phi(\xi)+\xi \phi^{\prime}(\xi)\right)-\frac{v_{-}}{1+v_{-}} \phi^{\prime}(\xi)\right) d \xi \\
& \quad+\int_{\xi_{\beta}}^{\xi_{2}}\left(v_{+}\left(\phi(\xi)+\xi \phi^{\prime}(\xi)\right)-\frac{v_{+}}{1+v_{+}} \phi^{\prime}(\xi)\right) d \xi=0 .
\end{aligned}
$$

Through simplifying (50), we get

$$
\phi\left(\xi_{\beta}\right) \cdot\left(v_{-} \xi_{\beta}-\frac{v_{-}}{1+v_{-}}-v_{+} \xi_{\beta}+\frac{v_{+}}{1+v_{+}}\right)=0,
$$

which leads to $\xi_{\beta}=1 /\left(1+v_{-}\right)\left(1+v_{+}\right)$for the arbitrary of $\phi(\xi)$.

Let us turn back to calculate $\xi_{\alpha}$; we have

$$
\begin{aligned}
\left(1+v_{+}\right) \xi_{\beta} & =\frac{1}{1+v_{-}} \geq \xi_{\alpha}=\lim _{\varepsilon \rightarrow 0^{+}} \xi_{\alpha}^{\varepsilon} \\
& =\lim _{\varepsilon \rightarrow 0^{+}} \frac{1}{1+v^{\varepsilon}\left(\xi_{\alpha}^{\varepsilon}\right)} \geq \frac{1}{1+v_{+}}=\left(1+v_{-}\right) \xi_{\beta} .
\end{aligned}
$$


It is clear that $1 /\left(1+v_{-}\right)>\xi_{\beta}>1 /\left(1+v_{+}\right)$for $-1<v_{-}<$ $0<v_{+}$. Furthermore, if we assume that $1 /\left(1+v_{-}\right)>\xi_{\alpha}>$ $\xi_{\beta}$, through taking $\eta=\left(\xi_{\alpha}-\xi_{\beta}\right) / 2>0$ here, then we can take $\varepsilon>0$ sufficiently small such that $\xi_{\alpha}^{\varepsilon}>\xi_{\alpha}-\eta=\xi_{\beta}+\eta$ for $\xi_{\alpha}=\lim _{\varepsilon \rightarrow 0^{+}} \xi_{\alpha}^{\varepsilon}$. It can be derived from Lemma 8 that $\lim _{\varepsilon \rightarrow 0^{+}} v^{\varepsilon}\left(\xi_{\alpha}^{\varepsilon}\right)=v_{+}$. Thus, we arrive at $\xi_{\alpha}=1 /\left(1+v_{+}\right)$by applying (52), which contradicts the assumption $1 /\left(1+v_{-}\right)>$ $\xi_{\alpha}>\xi_{\beta}$.

On the other hand, if we assume that $\xi_{\beta}>\xi_{\alpha}>1 /\left(1+v_{+}\right)$, by taking $\eta=\left(\xi_{\beta}-\xi_{\alpha}\right) / 2>0$ now, then we can also take $\varepsilon>0$ sufficiently small such that $\xi_{\alpha}^{\varepsilon}<\xi_{\alpha}+\eta=\xi_{\beta}-\eta$. It can be derived from Lemma 8 that $\lim _{\varepsilon \rightarrow 0^{+}} v^{\varepsilon}\left(\xi_{\alpha}^{\varepsilon}\right)=v_{-}$. Thus, we arrive at $\xi_{\alpha}=1 /\left(1+v_{-}\right)$by applying (52), which also contradicts the assumption $\xi_{\beta}>\xi_{\alpha}>1 /\left(1+v_{+}\right)$.

From the above discussion, we can get the relation $\xi_{\alpha}=$ $\xi_{\beta}=1 /\left(1+v_{-}\right)\left(1+v_{+}\right)$. The proof is completed.

Lemma 10. For any $\eta>0$, one can obtain

$$
\lim _{\varepsilon \rightarrow 0^{+}} w^{\varepsilon}(\xi)= \begin{cases}w_{-}, & \text {for } \xi<\xi_{\beta}-\eta \\ w_{+}, & \text {for } \xi>\xi_{\beta}+\eta\end{cases}
$$

uniformly in the above intervals.

The process of this proof is similar to that of Lemma 5.3 in [5], and thus we omit it.

Theorem 11. Let $-1<v_{-}<0<v_{+}$and let $\left(v^{\varepsilon}(\xi), w^{\varepsilon}(\xi)\right)$ be the solution of (23) and (24). Then, the limit of $\left(v^{\varepsilon}(\xi), w^{\varepsilon}(\xi)\right)$ as $\varepsilon \rightarrow 0$ is (17) with (18), namely, the corresponding Riemann solution of (1) and (2).

Proof. To prove it, we should consider the limit behavior of $w^{\varepsilon}(\xi)$ in the neighborhood of $\xi=\sigma=1 /\left(1+v_{-}\right)\left(1+v_{+}\right)$. We take $\phi(\xi) \in C_{0}^{\infty}\left(\xi_{1}, \xi_{2}\right)$ with $\xi_{1}<\sigma<\xi_{2}$ and satisfy $\phi(\xi)=$ $\phi(\sigma)$ on the neighborhood of $\xi=\sigma$ such as $N(\mu)=[\sigma-\mu, \sigma+$ $\mu$ ] for sufficiently small $\mu>0$, which is called the sloping test functions in [22].

Then, by (26), we have

$$
\begin{aligned}
& \int_{\xi_{1}}^{\xi_{2}}\left(-\xi w_{\xi}^{\varepsilon}+\left(\frac{w^{\varepsilon}}{1+v^{\varepsilon}}\right)_{\xi}\right) \phi(\xi) d \xi \\
& =\int_{\xi_{1}}^{\xi_{2}}\left(w^{\varepsilon} \phi+w^{\varepsilon}\left(\xi-\frac{1}{1+v^{\varepsilon}}\right) \phi^{\prime}\right) d \xi=0 .
\end{aligned}
$$

It follows from $\phi(\xi)=\phi(\sigma)$ for $\xi \in N(\mu)$ that

$$
\begin{aligned}
\lim _{\varepsilon \rightarrow 0^{+}} & \int_{\xi_{1}}^{\xi_{2}}\left(w^{\varepsilon}\left(\xi-\frac{1}{1+v^{\varepsilon}}\right) \phi^{\prime}\right) d \xi \\
= & \int_{\xi_{1}}^{\sigma-\mu}\left(w_{-}\left(\xi-\frac{1}{1+v_{-}}\right) \phi^{\prime}\right) d \xi \\
& +\int_{\sigma+\mu}^{\xi_{2}}\left(w_{+}\left(\xi-\frac{1}{1+v_{+}}\right) \phi^{\prime}\right) d \xi
\end{aligned}
$$

$$
\begin{aligned}
= & w_{-}\left(\sigma-\mu-\frac{1}{1+v_{-}}\right) \phi(\sigma) \\
& -w_{-} \int_{\xi_{1}}^{\sigma-\mu} \phi d \xi-w_{+}\left(\sigma+\mu-\frac{1}{1+v_{+}}\right) \phi(\sigma) \\
& -w_{+} \int_{\sigma+\mu}^{\xi_{2}} \phi d \xi .
\end{aligned}
$$

By taking the limit $\mu \rightarrow 0$ above, we have

$$
\begin{aligned}
\lim _{\varepsilon \rightarrow 0^{+}} & \int_{\xi_{1}}^{\xi_{2}}\left(w^{\varepsilon}\left(\xi-\frac{1}{1+v^{\varepsilon}}\right) \phi^{\prime}\right) d \xi \\
= & \left(-\sigma[w]+\left[\frac{w}{1+v}\right]\right) \phi(\sigma) \\
& \quad-\int_{\xi_{1}}^{\xi_{2}}\left(w_{-} H(\sigma-\xi)+w_{+} H(\xi-\sigma)\right) \phi(\xi) d \xi,
\end{aligned}
$$

in which $H$ denotes the Heaviside function.

Combining (54) together with (56), we have

$$
\begin{aligned}
& \lim _{\varepsilon \rightarrow 0^{+}} \int_{\xi_{1}}^{\xi_{2}}\left(w^{\varepsilon}-w_{-} H(\sigma-\xi)-w_{+} H(\xi-\sigma)\right) \phi(\xi) d \xi \\
& \quad=\left(\sigma[w]-\left[\frac{w}{1+v}\right]\right) \phi(\sigma)
\end{aligned}
$$

for all sloping test functions $\phi(\xi) \in C_{0}^{\infty}\left[\xi_{1}, \xi_{2}\right]$. With the same technique in $[5,22]$, one can see that the conclusion can also be drawn for any test function $\phi(\xi) \in C_{0}\left[\xi_{1}, \xi_{2}\right]$.

By taking the limits $\xi_{1} \rightarrow \sigma-$ and $\xi_{2} \rightarrow \sigma+$ in (57), we can obtain

$$
\begin{aligned}
w(\xi)= & \lim _{\varepsilon \rightarrow 0^{+}} w^{\varepsilon}(\xi) \\
= & w_{-} H(\sigma-\xi)+w_{+} H(\xi-\sigma) \\
& +\left(\sigma[w]-\left[\frac{w}{1+v}\right]\right) \delta(\xi-\sigma) .
\end{aligned}
$$

Thus the limit of $w^{\varepsilon}(\xi)$ is exactly the corresponding Riemann solution (17) with (18) for $w$ when $-1<v_{-}<0<v_{+}$.

Finally, the value of $v(\xi)$ at the discontinuity point $\xi=$ $\sigma$ should be calculated. Then, (26) can be changed into the following form:

$$
\int_{\xi_{1}}^{\xi_{2}} w \cdot\left(\phi+\xi \phi^{\prime}-\frac{\phi^{\prime}}{1+v}\right) d \xi=0 .
$$

By substituting (58) into (59), through a simple calculation, one can arrive at

$$
\begin{aligned}
& \int_{\xi_{1}}^{\sigma-}\left(\phi+\xi \phi^{\prime}-\frac{\phi^{\prime}}{1+v}\right) d \xi+\int_{\sigma+}^{\xi_{2}}\left(\phi+\xi \phi^{\prime}-\frac{\phi^{\prime}}{1+v}\right) d \xi \\
& \quad+\left(\sigma[w]-\left[\frac{w}{1+v}\right]\right)\left(\phi(\sigma)+\sigma \phi^{\prime}(\sigma)-\frac{\phi^{\prime}(\sigma)}{1+v_{\delta}}\right)=0 ;
\end{aligned}
$$


namely,

$$
\begin{aligned}
& w_{-}\left(\sigma-\frac{1}{1+v_{-}}\right) \phi(\sigma)-w_{+}\left(\sigma-\frac{1}{1+v_{+}}\right) \phi(\sigma) \\
& +\left(\sigma[w]-\left[\frac{w}{1+v}\right]\right)\left(\phi(\sigma)+\sigma \phi^{\prime}(\sigma)-\frac{\phi^{\prime}(\sigma)}{1+v_{\delta}}\right)=0 .
\end{aligned}
$$

Since $\phi$ is arbitrary, one can obtain $\sigma=1 /\left(1+v_{\delta}\right)=1 /(1+$ $\left.v_{-}\right)\left(1+v_{+}\right)$, which implies that $v_{\delta}=v_{-}+v_{+}+v_{+} v_{-}$.

\section{Acknowledgments}

This work is supported by the National Natural Science Foundation of China $(11001116,11271176)$ and the Project of Shandong Provincial Higher Educational Science and Technology Program (J11LA03, J12LI01) and Shandong Provincial Natural Science Foundation (ZR2010AL012).

\section{References}

[1] M. Mazzotti, "Non-classical composition fronts in nonlinear chromatography-delta-shock," Industrial \& Engineering Chemistry Research, vol. 48, pp. 7733-7752, 2009.

[2] M. Mazzotti, A. Tarafder, J. Cornel, F. Gritti, and G. Guiochon, "Experimental evidence of a delta-shock in nonlinear chromatography," Journal of Chromatography A, vol. 1217, pp. 20022012, 2010.

[3] H. Cheng and H. Yang, "Delta shock waves in chromatography equations," Journal of Mathematical Analysis and Applications, vol. 380, no. 2, pp. 475-485, 2011.

[4] V. M. Shelkovich, "Delta-shock waves in nonlinear chromatography," in Proceedings of the 13th International Conference on Hyperbolic Problems: Theory, Numerics, Applications, Beijing, China, 2010.

[5] M. Sun, "Delta shock waves for the chromatography equations as self-similar viscosity limits," Quarterly of Applied Mathematics, vol. 69, no. 3, pp. 425-443, 2011.

[6] H. Yang and Y. Zhang, "New developments of delta shock waves and its applications in systems of conservation laws," Journal of Differential Equations, vol. 252, no. 11, pp. 5951-5993, 2012.

[7] B. Temple, "Systems of conservation laws with invariant submanifolds," Transactions of the American Mathematical Society, vol. 280, no. 2, pp. 781-795, 1983.

[8] L. Ambrosio, G. Crippa, A. Figalli, and L. V. Spinolo, "Some new well-posedness results for continuity and transport equations, and applications to the chromatography system," SIAM Journal on Mathematical Analysis, vol. 41, no. 5, pp. 1890-1920, 2009.

[9] C. Shen, "Wave interactions and stability of the Riemann solutions for the chromatography equations," Journal of Mathematical Analysis and Applications, vol. 365, no. 2, pp. 609-618, 2010.

[10] M. Sun, "Interactions of elementary waves for the Aw-Rascle model," SIAM Journal on Applied Mathematics, vol. 69, no. 6, pp. 1542-1558, 2009.

[11] M. Sun, "Interactions of delta shock waves for the chromatography equations," Applied Mathematics Letters, vol. 26, no. 6, pp. 631-637, 2013.
[12] G. Wang, "One-dimensional non-linear chromatography system and $\delta$-shock waves," Zeitschrift für Angewandte Mathematik und Physik, vol. 64, no. 5, pp. 1451-1469, 2013.

[13] C. M. Dafermos, "Solution of the Riemann problem for a class of hyperbolic systems of conservation laws by the viscosity method," Archive for Rational Mechanics and Analysis, vol. 52, pp. 1-9, 1973.

[14] M. Slemrod and A. E. Tzavaras, "A limiting viscosity approach for the Riemann problem in isentropic gas dynamics," Indiana University Mathematics Journal, vol. 38, no. 4, pp. 1047-1074, 1989.

[15] C. M. Dafermos, "Admissible wave fans in nonlinear hyperbolic systems," Archive for Rational Mechanics and Analysis, vol. 106, no. 3, pp. 243-260, 1989.

[16] G. Ercole, "Delta-shock waves as self-similar viscosity limits," Quarterly of Applied Mathematics, vol. 58, no. 1, pp. 177-199, 2000.

[17] J. Hu, "A limiting viscosity approach to Riemann solutions containing delta-shock waves for nonstrictly hyperbolic conservation laws," Quarterly of Applied Mathematics, vol. 55, no. 2, pp. 361-373, 1997.

[18] J. Hu, "The Riemann problem for a resonant nonlinear system of conservation laws with Dirac-measure solutions," Proceedings of the Royal Society of Edinburgh A, vol. 128, no. 1, pp. 81-94, 1998.

[19] F. Huang, "Existence and uniqueness of discontinuous solutions for a hyperbolic system," Proceedings of the Royal Society of Edinburgh A, vol. 127, no. 6, pp. 1193-1205, 1997.

[20] J. Li and H. Yang, "Delta-shocks as limits of vanishing viscosity for multidimensional zero-pressure gas dynamics," Quarterly of Applied Mathematics, vol. 59, no. 2, pp. 315-342, 2001.

[21] W. Sheng and T. Zhang, "The Riemann problem for the transportation equations in gas dynamics," Memoirs of the American Mathematical Society, vol. 137, no. 654, 1999.

[22] D. C. Tan, T. Zhang, and Y. X. Zheng, "Delta-shock waves as limits of vanishing viscosity for hyperbolic systems of conservation laws," Journal of Differential Equations, vol. 112, no. 1, pp. 1-32, 1994.

[23] F. Bouchut, "On zero pressure gas dynamics," in Advances in Kinetic Theory and Computing, vol. 22, pp. 171-190, World Scientific Publishing, River Edge, NJ, USA, 1994.

[24] G.-Q. Chen and H. Liu, "Formation of $\delta$-shocks and vacuum states in the vanishing pressure limit of solutions to the Euler equations for isentropic fluids," SIAM Journal on Mathematical Analysis, vol. 34, no. 4, pp. 925-938, 2003.

[25] F. Huang and Z. Wang, "Well posedness for pressureless flow," Communications in Mathematical Physics, vol. 222, no. 1, pp. 117146, 2001.

[26] J. Li, T. Zhang, and S. Yang, The two-dimensional Riemann problem in gas dynamics, vol. 98 of Pitman Monographs and Surveys in Pure and Applied Mathematics, Longman Scientific and Technical, Harlow, UK, 1998.

[27] C. Shen and M. Sun, "Formation of delta shocks and vacuum states in the vanishing pressure limit of Riemann solutions to the perturbed Aw-Rascle model," Journal of Differential Equations, vol. 249, no. 12, pp. 3024-3051, 2010.

[28] L. Guo, W. Sheng, and T. Zhang, "The two-dimensional Riemann problem for isentropic Chaplygin gas dynamic system," Communications on Pure and Applied Analysis, vol. 9, no. 2, pp. 431-458, 2010.

[29] G. Lai, W. Sheng, and Y. Zheng, "Simple waves and pressure delta waves for a Chaplygin gas in two-dimensions," Discrete 
and Continuous Dynamical Systems A, vol. 31, no. 2, pp. 489523, 2011.

[30] V. G. Danilov and V. M. Shelkovich, "Delta-shock wave type solution of hyperbolic systems of conservation laws," Quarterly of Applied Mathematics, vol. 63, no. 3, pp. 401-427, 2005.

[31] B. T. Hayes and P. G. LeFloch, "Measure solutions to a strictly hyperbolic system of conservation laws," Nonlinearity, vol. 9, no. 6, pp. 1547-1563, 1996.

[32] B. L. Keyfitz and H. C. Kranzer, "Spaces of weighted measures for conservation laws with singular shock solutions," Journal of Differential Equations, vol. 118, no. 2, pp. 420-451, 1995.

[33] D. J. Korchinski, Solution of a Riemann problem for a system of conservation laws possessing no classical weak solution [thesis], Adelphi University, 1977.

[34] M. Nedeljkov, "Shadow waves: entropies and interactions for delta and singular shocks," Archive for Rational Mechanics and Analysis, vol. 197, no. 2, pp. 489-537, 2010.

[35] V. M. Shelkovich, "Singular solutions of $\delta$ - and $\delta$ '-shock wave type of systems of conservation laws, and transport and concentration processes," Russian Mathematical Surveys, vol. 63, pp. 473-546, 2008.

[36] V. G. Danilov and V. M. Shelkovich, "Dynamics of propagation and interaction of $\delta$-shock waves in conservation law systems," Journal of Differential Equations, vol. 211, no. 2, pp. 333-381, 2005.

[37] H. Kalisch and D. Mitrović, "Singular solutions of a fully nonlinear $2 \times 2$ system of conservation laws," Proceedings of the Edinburgh Mathematical Society II, vol. 55, no. 3, pp. 711-729, 2012. 


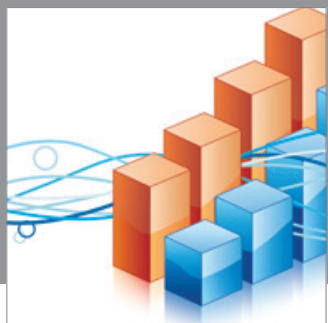

Advances in

Operations Research

mansans

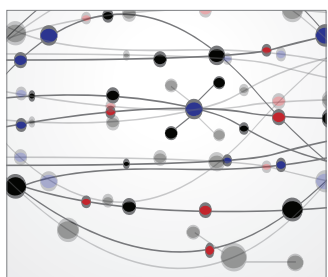

The Scientific World Journal
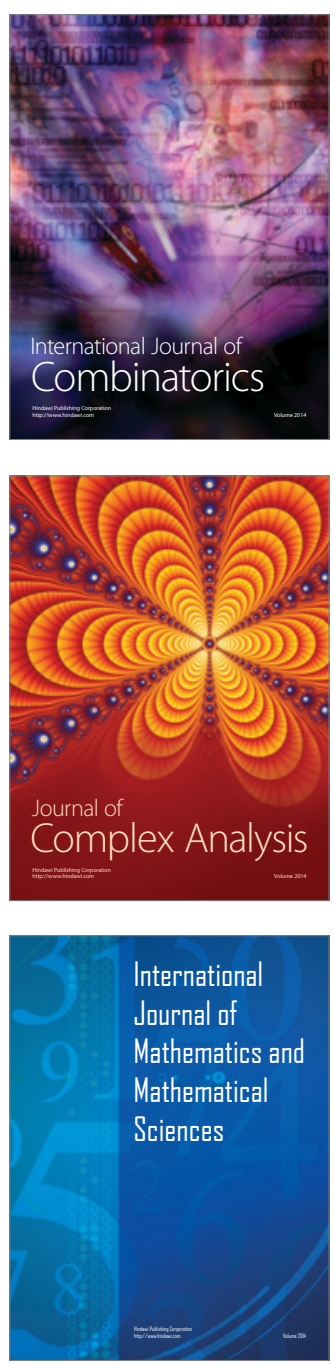
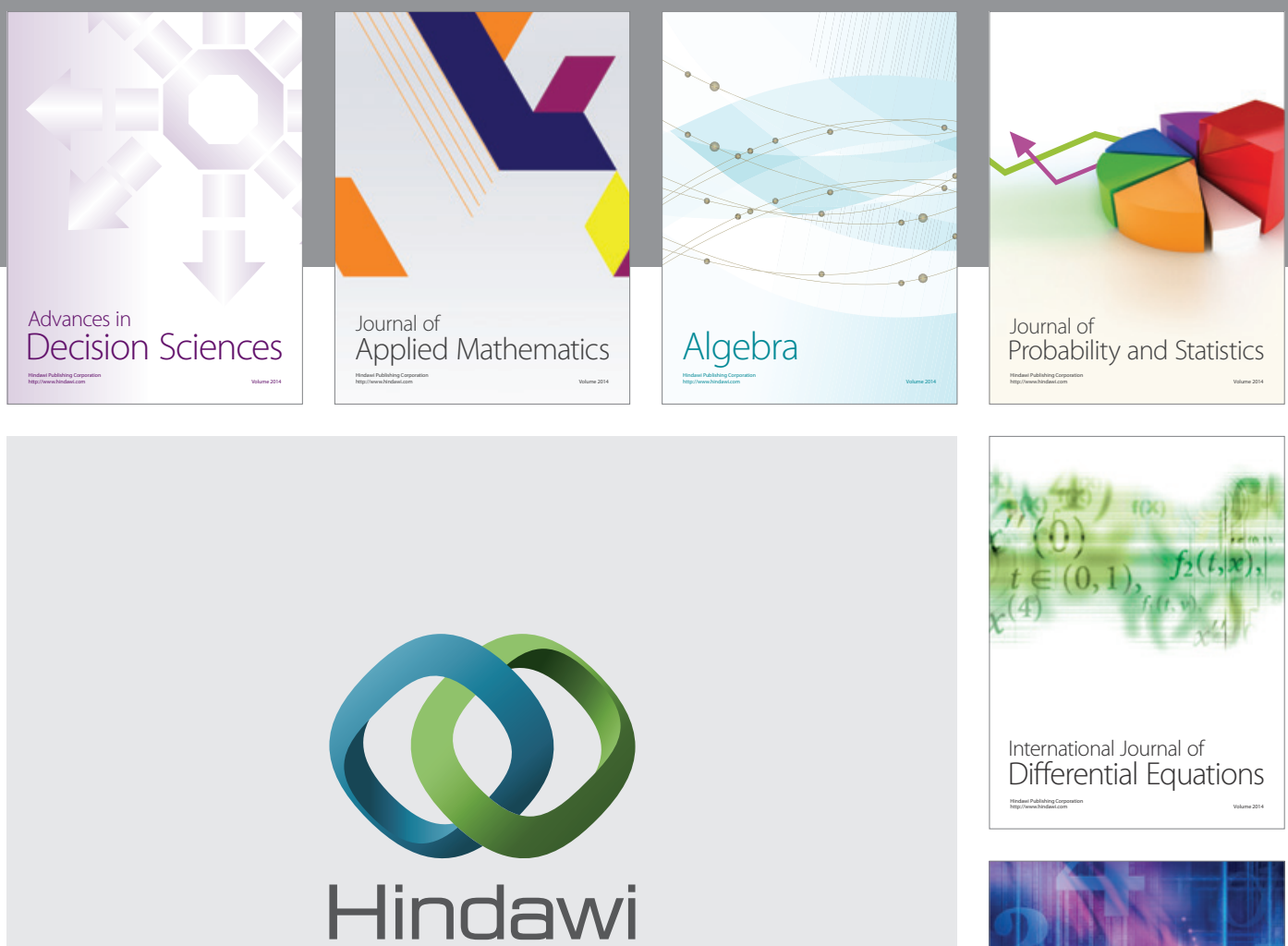

Submit your manuscripts at http://www.hindawi.com
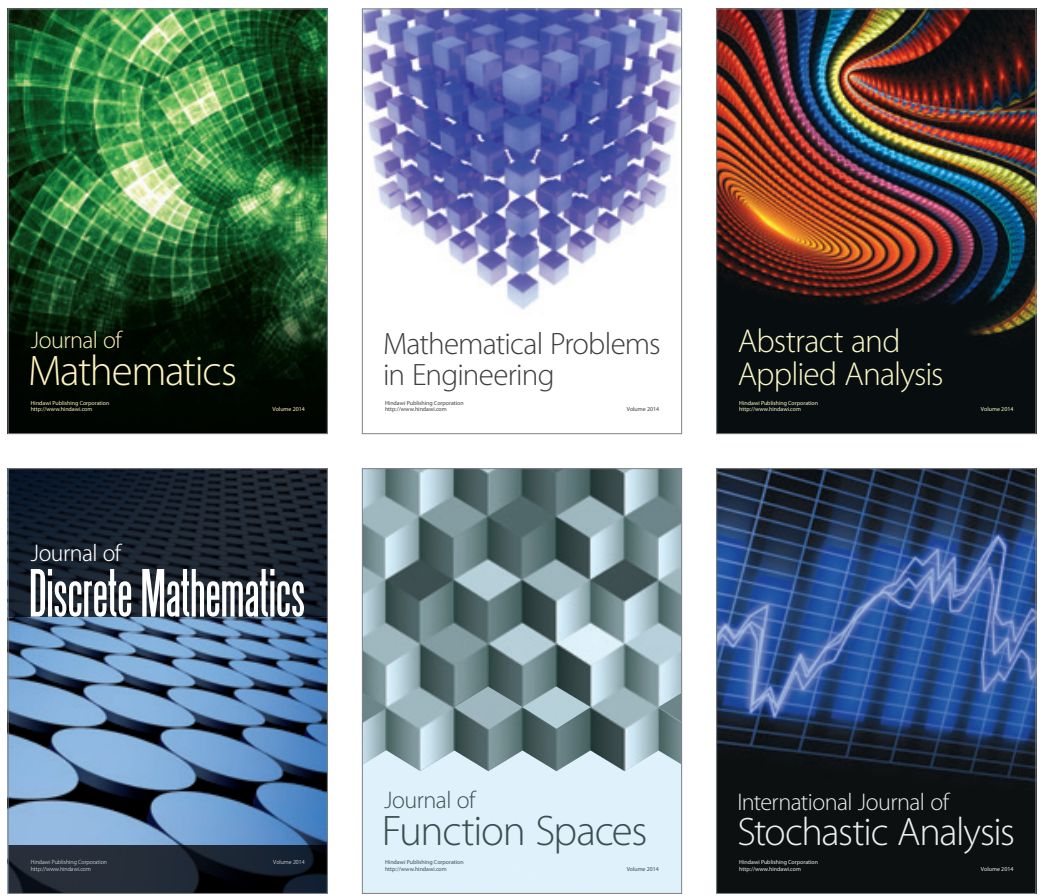

Journal of

Function Spaces

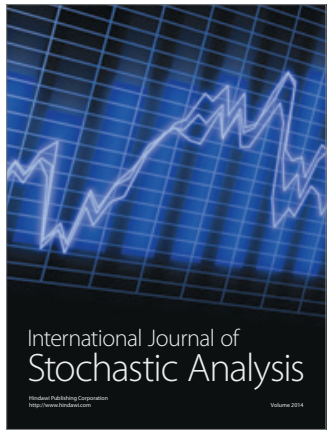

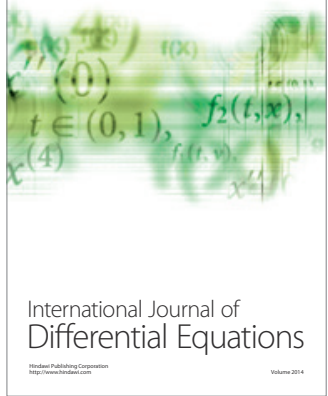
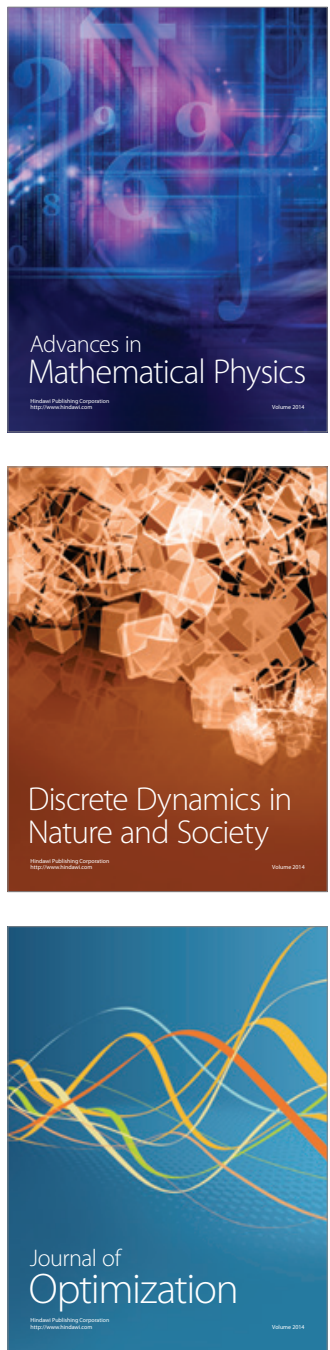